\title{
Respiratory rehabilitation techniques used for the treatment of COPD patients
}

\author{
PATRASCA Georgeta ${ }^{1}$, POPESCU Gilda ${ }^{2}$, TOFOLEAN Doina Ecaterina ${ }^{3}$, MAIEREAN Anca ${ }^{4}$, \\ DOGARU Gabriela ${ }^{4}$, CHIS Ana ${ }^{4}$, MOTOC Nicoleta Stefania ${ }^{4}$, FILDAN Ariadna Petronela ${ }^{1,3}$
}

Corresponding author: POPESCU Gilda, E-mail: gilda.popescu1958@gmail.com

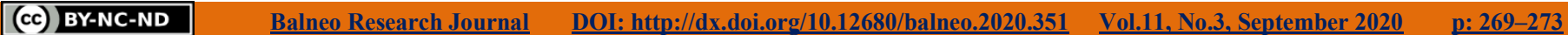

Peer reviewer: Sînziana Călina SILIȘTEANU, Romanian Association of Balneology, office@bioclima.ro

1. Clinical Pneumophtisiology Hospital, Constanta, Romania 2. “Titu Maiorescu” University, Faculty of Medicine, Bucharest, Romania

3. "Ovidius" University, Faculty of Medicine, Constanta, Romania

Abstract

4. "Iuliu Hatieganu" University of Medicine and Pharmacy, Cluj-Napoca, Romania

Introduction. Chronic obstructive pulmonary disease (COPD) is one of the leading causes of morbidity and mortality throughout the world. At present, the pharmacological therapy is not enough for a patient with COPD in regard to heath status and exercise tolerance. It requires additional therapies aimed to improve the quality of life, delay the lung function decline, increase exercise capacity, and reduce the respiratory symptoms and the number of exacerbations. Pulmonary rehabilitation program (PRP) has been shown to improve respiratory muscle, to optimize functional, psychosocial, behavioral and nutritional status. The aim of the present study was to asses the impact of PRP on COPD patients, focusing on the clinical benefits of PRP, which may further provide to the patients a good support for change. Material and method. Sixty-seven patients with clinically stable COPD were enrolled in this prospective study, consisting in a 6-weeks of PRP. There were recorded demographic data, smoking and medical history, and abnormal findings at the physical examination. All patients completed CAT (COPD Assessment Test) questionnaire and dyspnea on Borg scale, they performed spirometry, and six minutes walking test (6MWT), before and after the completion of the rehabilitation program. Results and Discussions. After the 6-week period of PRP, a large proportion of patients presented higher values of spirometric parameters, although the change was no statistically significant. The impact of COPD on the patient's health was significantly improved, $58.2 \%$ of patients registered $<10$ points of CAT questionnaire after PRP $(\mathrm{p}<0.05)$. 75\% of patients reported a 0-5 points of dyspnea on Borg scale comparing with $29 \%$ before the PRP ( $\mathrm{p}=0.0147$ ), and $56 \%$ a $0-5$ points of fatigue while only $25 \%$ of patients had the same score before the rehabilitation programme $(\mathrm{p}=0,022)$. Only 8 patients $(11.94 \%$ vs $31.34 \%, \mathrm{p}<0.05)$ reported a $\mathrm{SaO}_{2}$ lower than $90 \%$ after the PRP. The $6 \mathrm{MWD}$ was longer than $250 \mathrm{~m}$ at the end of the 6 -weeks of PRP for 38 patients $(56.7 \%$ vs $22.38 \%, \mathrm{p}<0.05)$. Conclusions. The present study showed that a PRP added to pharmacological treatment had a beneficial role of increasing the patient' health status and exercise tolerance for COPD patients.

Key words: $C O P D$, pulmonary rehabilitation, quality of life ( $Q o L)$,

\section{Introduction}

Chronic obstructive pulmonary disease (COPD) is one of the leading causes of morbidity and mortality throughout the world, representing a major public health problem (1-3). Tobacco smoking remains the main cause of COPD worldwide (4-7), but there are many other risk factors, such as biomass fuel, ambient particulate matter pollution or occupational exposure which may become more important in the future, especially in low- and middle-income countries $(8,9)$. Despite advances in pharmacological therapy, a significant proportion of patients continue to be symptomatic and suffer from repeated exacerbations and hospitalizations (10). In addition, COPD patients often associate concomitant chronic diseases that have an additive contribution to pregressive dyspnea and diminished tolerance to effort (11-14). Physical inactivity is a well-knowed factor strongly related to an impaired health status, hospital admission and mortality in COPD patients $(10,15)$. Thus, there is an obvious clinical indication for supplementary, comprehensive interventions such as pulmonary rehabilitation (PR), according to individual's characteristics and comorbidities.

Pulmonary rehabilitation is an effective nonpharmacological treatment strategy used to improve patient symptoms, exercise tolerance and quality of life (10). PR has also been shown to optimize functional, psychosocial, behavioral and nutritional status, increase participation, and reduce health care costs (16). The aim of the present study was to asses the impact of PR on COPD patients, focusing on the clinical benefits of $\mathrm{PR}$, which may further provide to the patients a good support for change. 


\section{Materials and methods}

The study was conducted at the Pulmonology Department of the Clinical Pneumophtisiology Hospital of Constanta, with the previous approval of the the ethical board. All patients signed the inform conset form before participating in the study. Sixtyseven patients with clinically stable COPD were enrolled in this prospective study, consisting in a 6weeks of pulmmonary rehabilitation program (PRP). All subjects were diagnosed with COPD and received pharmacological therapy according to GOLD criteria and to national recommendation of diagnosis and treatment of the COPD patients $(17,18)$. The inclusion criteria were: age of $40-75$ years, diagnosis of chronic symptomatic disease, no acute exacerbations within the 3 month, and eligibility to participate in exercise training. The exclusion criteria were: recent history of stroke or heart attack, neuromuscular disorders, orthopedic impairment, psychiatric illness or dementia, or uncontrolled chronic diseases under usual recommended therapy.

There were recorded demographic data, smoking and medical history, and abnormal findings at the physical examination. All patients completed CAT (COPD Assessment Test) questionnaire and dyspnea on Borg scale, they performed spirometry (Vitalograph ALPHA), and six minutes walking test (6MWT). CAT is a very simple, short and specific questionnaire that precisely assess the impact of COPD on the patient's health. It consists of 8 questions (each on a scale of 0 to 5 , with an overall score range of $0-40$ ) which cover various domains of respiratory health status (cough; sputum production; tightness in the chest; shortness of breath when walking upstairs; housework; ease of living at home; quality of sleep; energy) (19).

The 6MWT measures the distance that a patient can walk rapidly on a flat surface in a period of 6 minutes (6MWD). It evaluates the integrated responses of all the systems involved during walking, and for this reason, the 6MWD reflect the functional performance level for daily physical activities (20).

The PRP consisted in five sessions per week: a 30min education and exercises session, a 30-min of aerobic exercise session and a 20-min endurance exercise training (treadmill walking and/or stationary cycling on an ergo-metric bicycle, performed at $80 \%$ of patient's maximal heart rate). At the 30 -min aerobic exercise there was a 5-min warm-up period, followed by a 15-min of aerobic activity and a 10-min cool-down period. The aerobic activity included arm abduction into elevation and reverse, diagonal arm raises, arm abduction, forward flexion, and reverse, along with straight leg rises.

The PRP also included breathing exercises, stetching, along with education, smoking cessation intervention, nutritional intervention, and psychosocial support. The educational component comprised 12 sessions (twice a week) of discussions regarding the disease and the therapy, and practical demonstrations from a range of health care professionals (doctors, nurses and kinetotherapist).

Patients were reassessed using the same investigations after 6 weeks, at the end of the PRP.

All data were reported as means \pm standard deviation (SD) and a value of $p<0.05$ was accepted as a statistical significant change. Statistical analysis was performed using a statistical software program (GraphPad Prism 7).

\section{Results}

Sixty seven COPD patients of mean age 68.7 years, $55(82.08 \%)$ male, majority (85\%) with a smoking history of more than 20 pack/years, and more than a half with severe and very severe obstruction (FEV1< $50 \%$ ), were enrolled in to the six weeks of PRP. The baseline characteristics of the patients included in the study are listed in table 1.

Table 1. Baseline characteristics of the studied group

\begin{tabular}{|c|c|c|}
\hline Characteristics & $\mathrm{n}=$ & $\%$ \\
\hline Male/female gender & $55 / 12$ & $82.08 / 17.91$ \\
\hline Age (median, range) & \multicolumn{2}{|c|}{$68.7(44-82)$} \\
\hline $40-49$ & 2 & 2.98 \\
\hline $50-59$ & 10 & 14.92 \\
\hline $60-69$ & 32 & 47.76 \\
\hline$\geq 70$ & 23 & 34.32 \\
\hline Residence area (urban / rural) & $45 / 22$ & $67.15 / 32.77$ \\
\hline \multicolumn{3}{|l|}{ Smoking (pack/years) } \\
\hline$<20$ & 10 & 14.92 \\
\hline $20-40$ & 24 & 35.82 \\
\hline $40-60$ & 29 & 43.28 \\
\hline$>60$ & 4 & 5.97 \\
\hline \multicolumn{3}{|l|}{$B M I\left(\mathrm{~kg} / \mathrm{m}^{2}\right)$} \\
\hline Underweight $(<18.5)$ & 10 & 14.92 \\
\hline Normal weight $(18.5-25)$ & 24 & 35.82 \\
\hline Overweight $(25-30)$ & 25 & 37.31 \\
\hline Obese $(>30)$ & 8 & 11.94 \\
\hline \multicolumn{3}{|l|}{ COPD GOLD stage } \\
\hline $\mathrm{I}$ & 9 & 13.43 \\
\hline II & 20 & 29.85 \\
\hline III & 22 & 32.83 \\
\hline IV & 16 & 23.88 \\
\hline \multicolumn{3}{|l|}{ COPD phenotype } \\
\hline $\begin{array}{l}\text { Chronic } \\
\text { bronchitis/bronchiectasis }\end{array}$ & 40 & 59.7 \\
\hline Emphysema & 15 & 22.38 \\
\hline Asthma COPD overlap & 12 & 17.91 \\
\hline
\end{tabular}


After the 6-week period of PRP, a large proportion of patients presented higher values of spirometric parameters, although the change was no statistically significant, as shown in table 2 . The impact of COPD on the patient's health, measured by CAT questionnaire, was significantly improved, $58.2 \%$ of patients registered less than 10 points after PRP $(p<0.05)$. Dyspnea and fatigue were also considerably ameliorated at the end of the rehabilitation programme, $75 \%$ of patients reported a 0-5 points of dyspnea on Borg scale comparing with $29 \%$ before the PRP ( $\mathrm{p}=0.0147)$, and $56 \%$ a $0-5$ points of fatigue while only $25 \%$ of patients had the same score before the rehabilitation programme $(p=0,022)$. Only 8 patients $(11.94 \%$ vs $31.34 \%$, $\mathrm{p}<0.05)$ reported a $\mathrm{SaO}_{2}$ lower than $90 \%$ after the PRP. The $6 \mathrm{MWD}$ was longer than $250 \mathrm{~m}$ at the end of the 6 -weeks of PRP for 38 patients $(56.7 \%$ vs $22.38 \%, \mathrm{p}<0.05)$.

Table 2. Spirometric parameters and exercise assessments before and following PRP

\begin{tabular}{|c|c|c|c|c|c|}
\hline Parameters & \multicolumn{2}{|c|}{ Pre - PRP } & \multicolumn{2}{|c|}{ Post- PRP } & \multirow{2}{*}{$\begin{array}{l}\text { p value } \\
p=0.0765\end{array}$} \\
\hline$F E V_{1}(\%)$ & $\mathrm{n}=$ & $\%$ & $\mathrm{n}=$ & $\%$ & \\
\hline$>80$ & 9 & 13.43 & 11 & 16.41 & \\
\hline $50-80$ & 20 & 29.85 & 25 & 37.31 & \\
\hline $30-50$ & 22 & 32.83 & 24 & 35.82 & \\
\hline$<30$ & 16 & 23.88 & 7 & 10.44 & \\
\hline \multicolumn{5}{|c|}{$F E V_{1} / F V C(\%)$} & \multirow[t]{4}{*}{$\mathrm{p}=0.0681$} \\
\hline$>60$ & 20 & 29.85 & 33 & 49.25 & \\
\hline $45-60$ & 32 & 47.46 & 29 & 43.28 & \\
\hline$<45$ & 15 & 22.38 & 5 & 7.46 & \\
\hline \multicolumn{5}{|l|}{$C A T$} & \multirow[t]{4}{*}{$\mathrm{p}=0.0132$} \\
\hline$\leq 10$ & 19 & 28.35 & 39 & 58.20 & \\
\hline $10-20$ & 38 & 56.71 & 25 & 37.31 & \\
\hline$\geq 20$ & 10 & 14.92 & 3 & 4.47 & \\
\hline \multicolumn{5}{|c|}{ Dyspnea (Borg scale) } & \multirow[t]{5}{*}{$\mathrm{p}=0.0147$} \\
\hline $0-2$ & 5 & 7.46 & 15 & 22.38 & \\
\hline $3-5$ & 15 & 22.38 & 35 & 52.23 & \\
\hline $6-8$ & 37 & 55.22 & 15 & 22.38 & \\
\hline$\geq 9$ & 10 & 14.9 & 2 & 2.98 & \\
\hline \multicolumn{5}{|c|}{ Fatigue (Borg scale) } & \multirow[t]{5}{*}{$\mathrm{p}=0.022$} \\
\hline $0-2$ & 7 & 10.44 & 19 & 28.35 & \\
\hline $3-5$ & 18 & 26.86 & 37 & 55.22 & \\
\hline $6-8$ & 33 & 49.25 & 8 & 11.94 & \\
\hline$\geq 9$ & 9 & 13.43 & 3 & 4.47 & \\
\hline \multicolumn{5}{|l|}{$\mathrm{SaO}_{2}(\%)$} & \multirow[t]{6}{*}{$\mathrm{p}=0.0477$} \\
\hline$>98$ & 1 & 1.49 & 2 & 2.98 & \\
\hline $95-98$ & 10 & 14.92 & 12 & 17.91 & \\
\hline $92-95$ & 15 & 22.38 & 35 & 52.23 & \\
\hline $92-90$ & 20 & 29.85 & 10 & 14.92 & \\
\hline$<90$ & 21 & 31.34 & 8 & 11.94 & \\
\hline \multicolumn{5}{|l|}{$6 M W D(m)$} & \multirow[t]{5}{*}{$\mathrm{p}=0.0182$} \\
\hline$\leq 150$ & 16 & 23.88 & 8 & 11.94 & \\
\hline $150-250$ & 27 & 40.29 & 21 & 31.34 & \\
\hline $250-350$ & 19 & 28.35 & 27 & 40.29 & \\
\hline$\geq 350$ & 6 & 8.95 & 11 & 16.41 & \\
\hline
\end{tabular}

\section{Discussion}

The current study showed that a pulmonary rehabilitation program has significant clinical benefits for COPD patients, in regard to exercise tolerance and health status. Offering a comprehensive, patient-tailored, personalized intervention targeting multiple complex needs with the objective of improving physiological, social and psychological outcomes and to increase long-term adherence to health-enhancing behaviors must be the foundation of every PRP $(10,21)$.

The 6 weeks physical exercise program which was specially designed in order to increase the strength and endurance of the lower limb muscles by using a treadmill and/or an ergo-metric bicycle improved the exercise capacity and health status in patients with different COPD - GOLD stages and phenotypes. This beneficial results were also reported by other studies, with different duration of the PRP. Three to ten weeks of exercise-based PR has been shown to significantly impove not only the mainly symptoms (dyspnea and fatigue), but also the exercise capacity and quality of life in COPD patients, without a significant improvement in the degree of airflow obstruction (22-24).

Even if the CAT score is not precisely and uniquely linked to spirometric parameters and, therefore, it does not represent an alternative and reliable measurement to lung function, it emphasize important areas of the respiratory health-status in COPD patients, thus providing an valuable, simple, and objective tool for the long-term clinical and therapeutic assesing of COPD patients (19). This fact was also confirmed by the present study, which revealed a strong link among the CAT score and the respiratory symptoms reported by the patients, before and after the rehabilitation programme. Following the 6 weeks of PRP, three times less patients (3 of 67 patients, representing 4.47\%) had a CAT score $\geq 20$, while more than a half of them (39 of $67,58.2 \%$ ) scored less than 10 points of CAT questionnaire. Similar results were reported by the study of Dodd et al, in which were enrolled 261 patients with COPD participating in seven PR programmes. They reported a mean change in CAT score after PR of 2.9 (5.6) points, improving by 3.8 (6.1) points in those scoring ,,much better", and by 1.3 (4.5) in those who felt ,,a little better" $(p=0.002)$ (20). Onother study of 40 COPD patients showed a mean change in CAT score of $13(p<0.001)$, after the completion of $\mathrm{PR}$ programm. The change in CAT was significantly correlated with changes in 
Medical Research Council (MRC), St George's Respiratory Questionnaire (SRGQ) and London Chest Activity Daily Living (LCADL) scores, and Endurance Shuttle Walking Test (ESWT) (26).

One of the most important parameter used for monitoring COPD patients is represented by $\mathrm{SaO}_{2}$. It has been shown that daily activities, such as walking, washing and eating, are associated with transient oxygen desaturation in patients with moderate-to-severe COPD, even without marked resting hypoxaemia (27). The current study revealed a significant improvement in $\mathrm{SaO}$ after completing the PRP. Only 8 patients (11.95\%) had a $\mathrm{SaO}_{2}<90 \%$ at the end of the PRP, comparing to 21 patients $(31.34 \%, p<0.05)$ at the beginning of the study.

Similar results were reported by another study, in 2 . which the mean of $\mathrm{SaO}_{2}$ before rehabilitation was $93.8 \%$, and after PRP it was $97.16 \%$, with standard deviation of 0.645 and 0.374 respectively (28).

Patients with COPD usually complain of walking 3 . (gait) as representing one of the most difficult activity in daily life (29). Often, COPD patients walk less in daily life and cover shorter walk distances during the 6-min walk test (6MWT) comparing to healthy subjects (30). The present study has explored walking assessment in patients with COPD before and following the 6 weeks period of pulmonary rehabilitation programme, in order to assess their functional status. Thus, our results showed that more than a half of patients (56.7\%) reported an increase in the distance covered in the $6 \mathrm{MWT}$ at the end of the rehabilitation program; this changes may have been due to improvements in ventilatory capacity and also in respiratory patterns. One retrospective study of 245 patients with stable COPD who performed two 6MWT (one at enrollment and another after the completion of PRP) reported that $6 \mathrm{MWD}$ increased on the second test (all $\mathrm{p}<0.001$ ). At least $80 \%$ of patients walked 7 . further $(37 \mathrm{~m}, 95 \% \mathrm{CI}: 33-41 \mathrm{~m})$ on their second 6MWT (31).

The present study added supplemental information to the knowledge in regard to the benefits of the PRP on exercise capacity and the health status of COPD patients. It supports previous published data that a pulmonary rehabilitation program can provide significant clinical benefits to patients with COPD. This study was not designed to determine whether pulmonary rehabilitation programme reduces exacerbation rate and/or hospital admissions in COPD patients. Further studies should be performed in order to clear up these questions.

\section{Conclusions}

This study demonstrated that a 6-week pulmonary rehabilitation program achieved a clinically significant increase in patient' health status, and in exercise tolerance, even that the increase reported in spirometric parameters was not statistically significant.

Thus, adding a program of rehabilitation to the pharmacological therapy may represent another valuable tool in management of COPD patients.

\section{References}

1. Quaderi SA, Hurst JR. The unmet global burden of COPD. Glob Health Epidemiol Genom. 2018;3: e4.

2. Alexescu TG, Maierean A, Ciumarnean L, Budin C, Dogaru G, Todea DA. Rehabilitation therapies in stable chronic obstructive pulmonary disease. Balneo Research Journal. 2019;10(1):37-44.

3. Alexescu TG, Tarmure S, Negrean V, Cosnarovici M, Ruta VM, Popovici I, Para I, Perne MG, Orasan OH, Todea DA. Nanoparticles in the treatment of chronic lung diseases. J Mind Med Sci. 2019; 6(2): 224-231.

4. Rabe KF, Watz H. Chronic obstructive pulmonary disease. Lancet. 2017;389:1931-40.

5. Budin CE, Alexescu TG, Bordea IR, Gherginescu MC, Aluas M, Grigorescu BC, Biro L, Buzoianu AD, Nemes RM, Tantu MM, Todea DA. Nicotine Addiction Objective in Educational Programs for Smoking Prevention in Young People. REV.CHIM.(Bucharest).2019; 70(6): 2168- 72.

6. Budin C.E., Marginean C, Bordea I.R., Enache L.S., Enache E.L., Grigorescu B.L., et al. The Influence of Smoking on Nicotine Exposure Biomarkers and Inflammatory Profile Among Foster Care Teenagers. Rev.Chimia. 2018;69(12):3659-63.

7. Vremaroiu-Coman A, Alexescu TG, Negrean V, Milaciu MV, Buzoianu AD, Ciumarnean L, Todea DA. Ethical aspects of smoking cessation among the population from Transylvania. Balneo Research Journal. 2018;9(3):254-9.

8. Mannino DM Buist AS. Global burden of COPD: risk factors, prevalence, and future trends. Lancet. 2007;370:765-773.

9. Global, regional, and national comparative risk assessment of 79 behavioural, environmental and occupational, and metabolic risks or clusters of risks, 1990-2015: a systematic analysis for the Global Burden of Disease Study 2015. Lancet. 2016;388: 1659-724. 
10. Wouters E, Posthuma R, Koopman M, Liu WY, Sillen MJ, Hajian B, et al. An update on pulmonary rehabilitation techniques for patients with chronic obstructive pulmonary disease. Expert Review of Respiratory Medicine. 2020;14:2, 149-161.

11. Tofolean D, Popescu G, Arghir IA, Frandes M, Fildan AP. A Different Aproach of Chronic Obstructive Pulmonary Disease Severity and Plastic Medical Devices Used for Oxygenotherapy. Materiale Plastice. 2019; 56(2):295-300

12. Vanfleteren LE, Spruit MA, Groenen M, et al. Clusters of comorbidities based on validated objective measurements and systemic inflammation in patients with chronic obstructive pulmonary disease. Am J Respir Crit Care Med. 2013;187(7):728-735.

13. Deacu M, Tofolean DE, Bosoteanu $M$. Pulmonary alveolar lipoproteinosis associated with emphysematous areas. Rom J Morphol Embryol 2012;53(1):173-177

14. Trenchea M, Arghir IA, Popescu G, Rascu S, Bechir ES, Tofolean D, et al. The Triad Nocturia, Smoking and Obstructive Sleep Apnea. Rev Chim (Bucharest) 2019;70(5):1839-1842.

15. Waschki B, Kirsten A, Holz O, et al. Physical activity is the strongest predictor of all-cause mortality in patients with COPD: a prospective cohort study. Chest. 2011;140: 331-42.

16. Hopkinson N. Pulmonary Rehabilitation for COPD. Tanaffos. 2017;16(Suppl 1):S7-S8.

17. Global Initiative for Chronic Obstructive Lung Disease. Global strategy for the diagnosis, management and prevention of chronic obstructive pulmonary disease. 2019 Report.

18. Ulmeanu R, Fildan AP, Oancea C, Mihaltan F et al. Recomandari de diagnostic si tratament in bronhopneumopatia obstructiva cronica. Ed Medicala, Bucuresti, 2019.

19. Dal Negro, R.W., Bonadiman, L. \& Turco, P. Sensitivity of the COPD assessment test (CAT questionnaire) investigated in a population of 681 consecutive patients referring to a lung clinic: the first Italian specific study. Multidiscip Respir Med. 2014; 9: 15.

20. ATS Statement Guidelines for the Six-Minute Walk Test. Am J Respir Crit Care Med. 2002;166:111-117.

21. Maierean A, Ciumarnean L, Alexescu TG, Domokos B, Rajnoveanu R, Arghir O, Todea D,
Buzoianu AD, Dogaru G, Bordea RI. Complementary therapeutic approaches in asthma. Balneo Research Journal. 2019;10(3):204-212.

22. Houben-Wilke S, Augustin IM, Vercoulen JH, et al. COPD stands for complex obstructive pulmonary disease. Eur Respir Rev. 2018;27:148.

23. Spruit MA, Augustin IM, Vanfleteren LE, et al. Differential response to pulmonary rehabilitation in COPD: multidimensional profiling. Eur Respir J. 2015;46(6):1625-1635.

24. Janssen DJ, Wilke S, Smid DE, et al. Relationship between pulmonary rehabilitation and care dependency in COPD. Thorax. 2016;71(11):1054-1056.

25. Dodd JW, Hogg L, Nolan J, et alThe COPD assessment test (CAT): response to pulmonary rehabilitation. A multicentre, prospective studyThorax. 2011;66:425-429.

26. Candemir I, Kaymaz D, Ergun P, Demir N, Egesel N, Sengul F. Assessment of pulmonary rehabilitation efficacy in chronic obstructive pulmonary disease patients using the chronic obstructive pulmonary disease assessment test, Expert Review of Respiratory Medicine. 2015; 9(4): 487-492.

27. Schenkel N, Burdet L, Muralt B, Fitting JW. (1996). Oxygen saturation during daily activities in chronic obstructive pulmonary disease. Eur Respir J. Official Journal of the European Society for Clinical Respiratory Physiology. 1996;9: 2584-9.

28. Shanmugananth E, Kalindi D, Nambi G. Effect of Short Term Pulmonary Rehabilitation on Saturation of Peripheral Oxygen, Forced Vital Capacity, Forced Expiratory Volume in One Second and Exercise Capacity in Moderate COPD. International Journal of Allied Practice, Research and Review. 2015;2(9):01-12.

29. Annegarn J, Meijer K, Passos VL, et al. Problematic activities of daily life are weakly associated with clinical characteristics in COPD. J Am Med Dir Assoc. 2012;13(3):284-290.

30. Annegarn J, Spruit MA, Savelberg HH, et al. Differences in walking pattern during 6-min walk test between patients with COPD and healthy subjects. PloS One. 2012;7(5):e37329.

31. Jenkins S, Cecins NM. Six-minute walk test in pulmonary rehabilitation:do all patients need a practice test? Respirology. 2010;15(8):1192-6. 